\title{
Comparative analysis of cellulose pressboard and aramid paper used in air insulation systems of high-voltage devices
}

\author{
Tomasz Turba ${ }^{1, *}$, and Pawet Frącz ${ }^{1}$ \\ ${ }^{1}$ Opole University of Technology, Faculty of Electrical Engineering, Automatic Control and Computer Science, Prószkowska 76, \\ 45-758 Opole, Poland
}

\begin{abstract}
The paper presents results of a comparative analysis of parameters of two kinds of solid dielectrics used in air insulation systems to prevent occurring partial discharges. The research works regarded materials made of: cellulose pressboard and aramid paper. All measurements were performed under laboratory conditions by changing the value of partial discharges generation voltage until breakdown occurred in the inhomogeneous environment that was simulated using needle-plate (made of copper) electrode system. The main contribution which resulted from studies is a statement that potential use of aramid paper as a dielectric can extend the life of a high voltage electric device as compared to standard cellulose pressboard usage due to higher electric resistances to breakdown or detection of corona voltage. Results shown that the aramid paper has greater electric resistance to breakdown in comparison to cellulose with no difference between both on detecting corona of partial discharge.
\end{abstract}

\section{Introduction}

The research concerns a field of science which is related to generation of electric discharges on surfaces of highvoltage (HV) air insulation systems. Important problem is ageing process which progress during operation of equipment causing deterioration of insulating properties. Main ageing factors include: UV radiation, temperature fluctuations, ozone and nitrogen oxides, rime deposition and partial discharges (PD) [1]. Natural insulation of most air insulation systems provides atmospheric air having dielectric strength equal to $32 \mathrm{kV} / \mathrm{cm}$ in normal conditions. High voltage lines (e.g. $110 \mathrm{kV}$ ), distributions line along with power plants with substations form the key elements of a power system, which purpose is to produce and transmit energy to consumers. Interruptions in electricity supply can lead to financial losses, so all components of the power system should work reliably, safe and at the lower possible cost. Thus, to improve the dielectric strength of high voltage equipment it is necessary to use barriers of solid dielectric materials by placing them between electrodes in perpendicular or parallel manner [2]. The main objective of research conducted within this scope is to compare insulating properties to $\mathrm{PD}$ resistance of cellulose pressboard and aramid paper to determine as accurately as possible with the lowest installation cost whether aramid paper could succeed cellulose in normal operations. The studies involved measurements of electrical discharges occurring on the surface of both dielectrics. Based on the gathered data the initial voltage and breakdown values were estimated and compared to confirm the hypothesis.

\section{Materials and methodology for comparative analysis}

\subsection{Metrological parameters of the measuring system}

There are many measurement methods of detecting partial discharges occurring in insulation systems of electrical power machines. Methods can be divided into two groups: invasive and non-invasive methods. Invasive methods can detect and record PD currents with help of measuring probes expressing the PD value. This involves shutting off power supply for the device during connection/disconnection of probes which is a huge disadvantage [3]. Non-invasive methods include: acoustic emission [4,5], optical emission [6,7], electromagnetic or radio-frequency detection $[8,9]$. This paper concerns the use of invasive, classical method. Fig. 1 presents circuit diagram of measuring system using the experimental methodology which involved construction of a needle-plate electrodes system supplied by a $110 \mathrm{kV}$ test transformer to simulate inhomogeneous environment. Between the needle and the plate a double bakelite plate with a circular hole of diameter $s$ equal to $30 \mathrm{~cm}$ inside is placed. Between the plates the dielectric sample is installed as can be seen on Fig. 2. Consecutive measuring of electric strength is done by drifting apart the distance $a[\mathrm{~cm}]$ between dielectric sample and edge electrode.

Next step after breakdown is to extend distance from plate electrode to new sample series $(d \quad[\mathrm{~cm}])$. Incrementation by $1 \mathrm{~cm}$ after breakdown voltage is set

\footnotetext{
Corresponding authors: tturba@gmail.com
} 
for extending the distance from the edge electrode with constant value of $d$ for a new series of measurements.

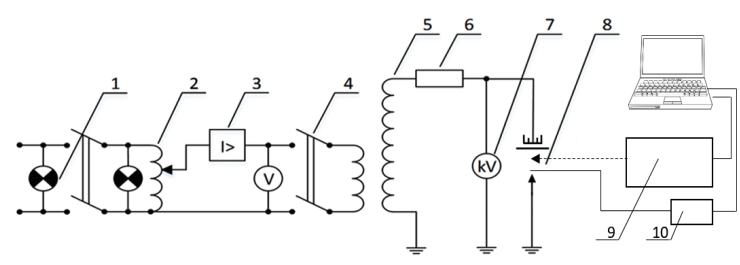

Fig. 1. Circuit diagram of the measuring system: 1 - pilot light, 2 - voltage regulator, 3 - overcurrent protection, 4 - circuit breaker, 5 - test transformer, 6 - protective resistor, 7 electrostatic voltmeter, 8 - edge-plate configuration.

Next, the distance $d$ is incremented by $1 \mathrm{~cm}$ and new measurement series is processed with extending the needle electrode $1 \mathrm{~cm}$ for each trial beginning again from $1 \mathrm{~cm}$. Three measurements for each distance were processed due to increase the probability of correct results. Each voltage for distance is a sum of arithmetic average value.

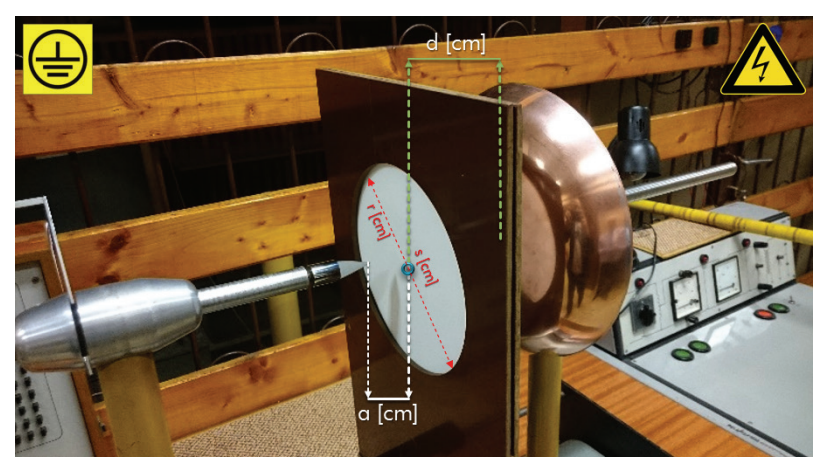

Fig. 2. Measurement system (needle-plate configuration) with aramid paper installed. Distances $a, d, s$ diameter and $r$ radius are shown.

The distance $a$ is in range from 1 to $15 \mathrm{~cm}$ and the distance $d$ from sample to plate varies from 1 to $12 \mathrm{~cm}$. When the distance $a$ is fully measured (every centimetre had its breakdown voltage value measured), the $d$ is being incremented by $1 \mathrm{~cm}$. If the breakdown voltage of $110 \mathrm{kV}$ power transformer cannot be achieved on a particular distance $a$, the measurement is finished and the distance $d$ is also incremented (distance $a$ after that is restarted from $1 \mathrm{~cm}$ for new group of measurement). The algorithm is shown in Fig. 3. In the research besides breakdown voltage, value also measured was corona voltage to verify whether the material type and parameters has any influence for observing phenomena. The Uo measurement took place during the measurement of $U \mathrm{~b}$ by generating voltage from $0 \mathrm{~V}$ to nearest possible visual descriptor seen by human eye in darkened lab room which corresponds to a value shown by electrostatic voltmeter. The value is being saved to database, and the generation of higher voltage continues until breakdown is achieved. It is possible to measure earlier (lower voltage value) and more accurate by using UV corona discharge camera like Ofil DayCor SuperB or spectrophotometer [6,7]. Using the OE method is part of future research of this study.

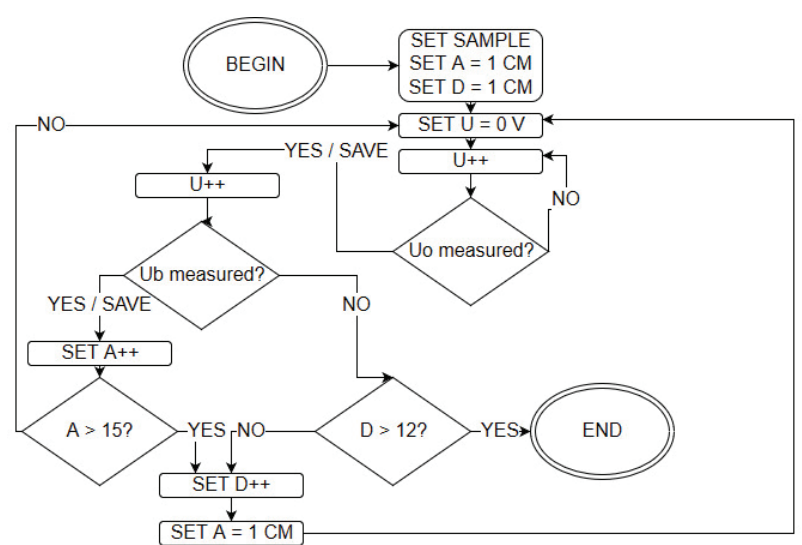

Fig. 3. Scheme of research algorithm of measuring $U o$ and $U \mathrm{~b}$ with increasing values of $a$ and $d$ distances in correct order.

\subsection{Characteristic of materials applied in the study}

Dielectrics can be classified according to different criteria. The most commonly used is the division from point of view of chemical properties. Therefore, there are three main categories: non-organic (e.g. glass, ceramic), organic (e.g. cellulose, resin) and polymers (e.g. aramid paper, polyvinyl chloride) [10]. Pressboard is a type of pressed cellulose cardboard produced by wet felting of vegetable fiber and flattening. For cellulose preparation, there is a need to apply two processes: acidic and alkaline, where it is less damaging for chemical molecules. Pressboard cellulose can be simply formed, trimmed or crushed so it is a commonly used material for insulating electrical conductors and transformers of high power [10]. It is a composite material like paper with the variation of sheet thickness ranged from $0.1 \mathrm{~mm}$ to plates of $50 \mathrm{~mm}$. It is characterized as highly resistant to discharge and can withstand short-term thermal load up to $350^{\circ} \mathrm{C}$.

The aramid paper is a polymer from the group of amides used in the production of fiber and sheets having both high mechanical strength and thermal resistance. It is made from synthetic fiber belonging to a group of polymers having amide linkages. The characteristic feature is existence of aromatic moieties in their main chains. If there is more in the structure of moieties, the greater mechanical strength, heat and fire resistance applies, but decreases the solubility (difficult in processing - more expensive). The material is akin to Kevlar in view of preparation by polymerizing 1,3diaminobenzene and isophthalic acid [11]. Yarns of aramid fibbers are formed by performing the wet liquid crystalline polymer solution in sulfuric acid. The fibbers are pressed into the slot with blowing air and then immersed in the bath to solidify. Sheets are produced on a paper machine sieve with a flat head by combining fibrids and flocs in aqueous solution. Finally, it is subjected to hot calendering giving it higher density resulting better mechanical and electrical properties [11].

Both cellulose pressboard and aramid can withstand high temperature impact and are similar in winding on a transformer coil of high power. Thus, they can be 
compared with the difference of cost. The aramid is far more expensive to produce than cheap cellulose, but initial research shows [12] that if probability of prolonging life of a high power equipment with aramid as insulation is greater than the cellulose - the risk of investment could be returned. The damage or investigation of shortcoming fault can be far more expensive in the mean of years of exploitation [13].

Materials used in research are both $0.1 \mathrm{~mm}$ thick and the size is $40 \mathrm{x} 40 \mathrm{~cm}^{2}$. The cellulose is from Tetrans company, model N125 and aramid paper is Nomex DuPont, model 410. When the sample is outdone after electrical breakdown, it is changed to a new, fresh one without changes in chemical structure because of shortthermal activity and ozone influence.

\section{Comparative analysis of measurement results}

Figs. 4-8 presents quadratic polynomial curve fitting models of aggregated values of $U o$ corona voltage, $U b$ breakdown voltage for cellulose and aramid papers.

It was respectively measured according to algorithm from Fig. 3 for various distances between electrodes $a[\mathrm{~cm}]$ and constant value of distance to dielectric barrier $d[\mathrm{~cm}]$.

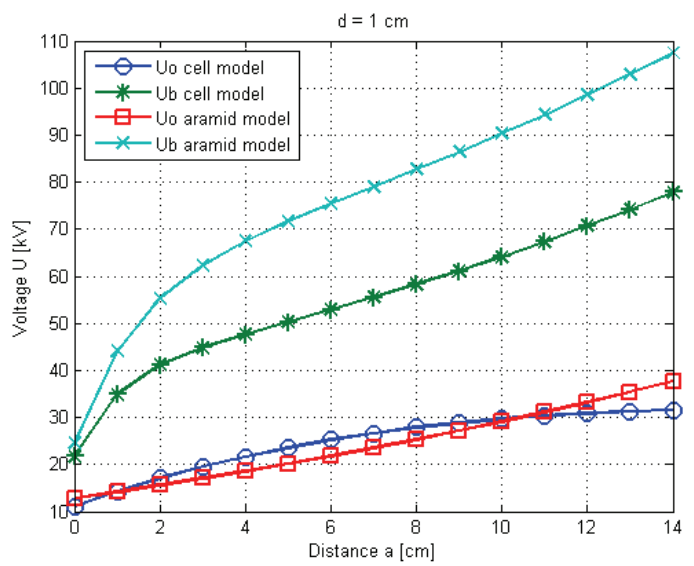

Fig. 4. Dependency of the voltage $U \mathrm{o}$ and $U \mathrm{~b}$ to the distance $a$ $1 \mathrm{~cm} \div 14 \mathrm{~cm}$ between electrodes at the constant distance $d=1$ $\mathrm{cm}$ for both materials.

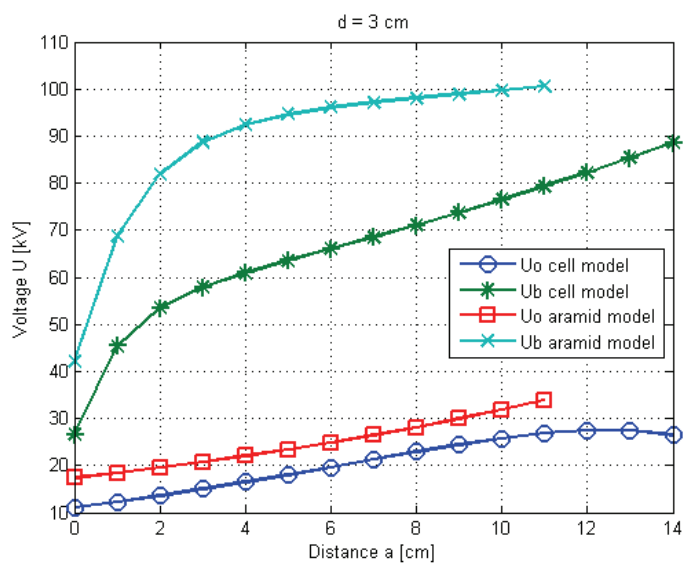

Fig. 5. Dependency of the voltage $U \mathrm{o}$ and $U \mathrm{~b}$ to the distance $a$ $1 \mathrm{~cm} \div 14 \mathrm{~cm}$ between electrodes at the constant distance $d=3$ $\mathrm{cm}$ for both materials.

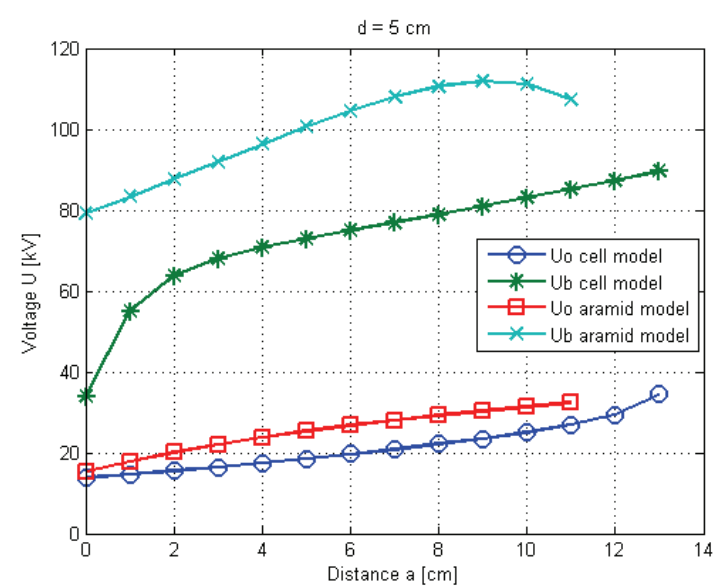

Fig. 6. Dependency of the voltage $U \mathrm{o}$ and $U \mathrm{~b}$ to the distance $a$ $1 \mathrm{~cm} \div 14 \mathrm{~cm}$ between electrodes at the constant distance $d=5$ $\mathrm{cm}$ for both materials.

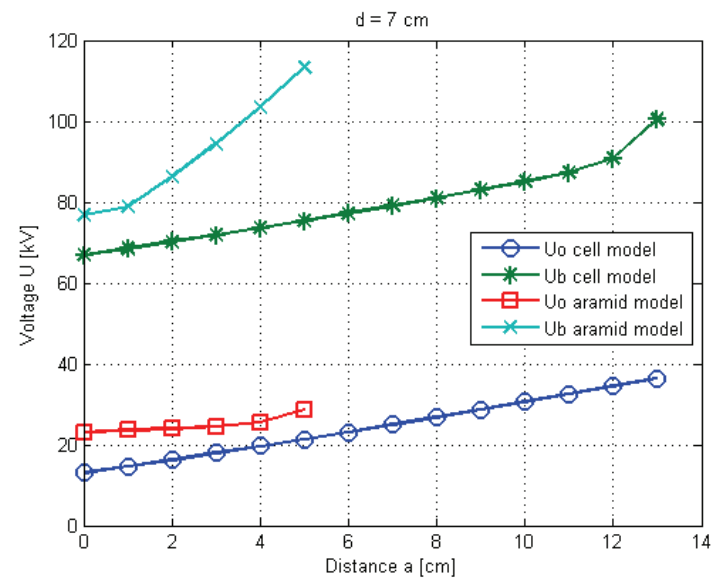

Fig. 7. Dependency of the voltage $U \mathrm{o}$ and $U \mathrm{~b}$ to the distance $a$ $1 \mathrm{~cm} \div 14 \mathrm{~cm}$ between electrodes at the constant distance $d=7$ $\mathrm{cm}$ for both materials.

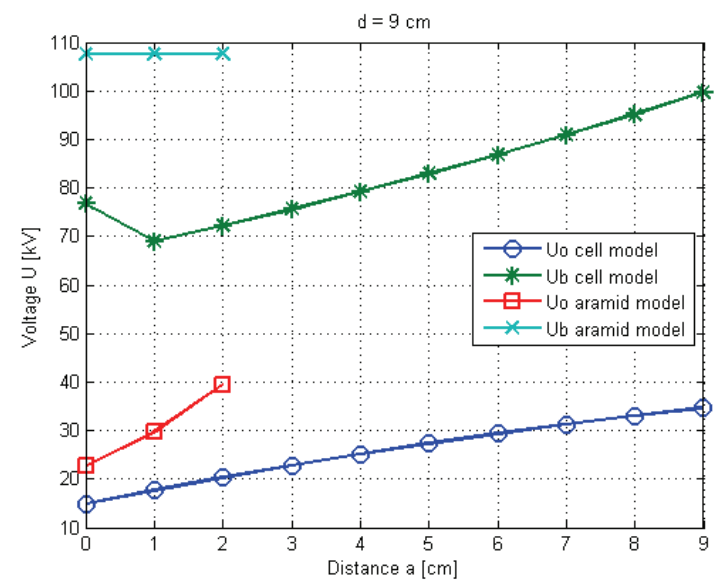

Fig. 8. Dependency of the voltage $U$ o and $U \mathrm{~b}$ to the distance $a$ $1 \mathrm{~cm} \div 14 \mathrm{~cm}$ between electrodes at the constant distance $d=9$ $\mathrm{cm}$ for both materials.

\section{Discussion of analysis results}

Based on the analysis, it was conducted that the measured corona voltages values has a low diversity coefficient value to assess that aramid paper is more 
resistant dielectric compared to cellulose paper. In the opposition stands the case of breakdown voltage, where the high difference was shown, when used various barriers as insulation. Furthermore, under laboratory environment, the research must have been finished when the aramid paper dielectric barrier of thickness $0.1 \mathrm{~cm}$ could not be penetrated. Voltage of $110 \mathrm{kV}$ was maximum possible value for transformer to generate on the power line connected to plate electrode. As can be seen on Figs. 4-6, the corona voltage $U$ o of barriers are in similar range of values. On Fig. 7 the $U$ o value for aramid paper is measured only when there was an electric penetration of barrier through generation of breakdown short-circuit. The lowest possible to measure value of corona voltage for cellulose pressboard of thickness $0.1 \mathrm{~cm}$ was $12.33 \mathrm{kV}$. In case of aramid paper of thickness $0.1 \mathrm{~cm}$ the lowest corona voltage value was $12.87 \mathrm{kV}$. The highest possible to measure value of corona voltage for cellulose pressboard was $23.90 \mathrm{kV}$ and $28.77 \mathrm{kV}$ for aramid paper respectively. The maximum possible distance between electrodes to create a breakdown voltage with cellulose was $12 \mathrm{~cm}$ which had given the full spectrum of measurement as was assumed for study. Aramid paper was far more resistant to short-circuit, thus the distance was lower in comparison $-9 \mathrm{~cm}$ with only two possible measurement distances between electrodes $(a=2 \mathrm{~cm})$. Power transformer of $110 \mathrm{kV}$ electric voltage was limited to destroy the sample with higher breakdown voltage value. Value of corona voltage increases almost linearly for both dielectric types. Value of breakdown voltage increases gradually with the increase of corona voltage for the lower values. However, this is not a linear relation. The distance between electrodes is directly proportional to higher voltage values generated in both dielectric types. Research have shown that aramid paper is far more resistant to electric breakdown in comparison to cellulose paper of same thickness. For PD detection purposes to observe $U_{0}$ it has no significance. Both values of corona voltage of PD on different materials are almost the same irrespectively which material was used.

\section{Conclusions}

Results of comparative analyses of corona and breakdown voltage in relation to the distance between $\mathrm{HV}$ and ground electrodes were presented in this paper. In particular two kinds of solid dielectrics used as barriers in air insulation of high voltage systems were considered: pressboard cellulose and aramid paper. The measured relations were registered under laboratory conditions using electrostatic voltmeter - in order to determine the influence of additional parameters as e.g. the distance between electrodes and the barrier, on the achieved results. The conducted analysis showed differences in values of corona and breakdown discharge occurrence for different solid dielectrics. The results obtained in the studies on aramid paper may help to improve diagnostics of power equipment, and succeed cellulose as a universal, insulation material for e.g. dry transformers (if risk of investment is calculated). In the future work, we aim to perform similar measurements for higher voltage values and for various thicknesses of the barrier. For corona discharge measurement it could be observed by using special UV spectrum camera. It has better accuracy than human eye and results can be obtained quicker on lower voltage value where human cannot see the UV emission of flowing electrons [6].

\section{References}

1. M. Kozioł, D. Wotzka, T. Boczar, P. Frącz, J. Spectro. 2016 (2016)

2. Ch. Liu, W. Zhang, M. Xu, L. Shi. Y. Zhao, IEEE ICD, 2, 772-775 (2016)

3. P. Frącz, T. Boczar, D. Zmarzły, T. Szczyrba, Acta Phys. Pol. A 124, 413-416 (2013)

4. H. Majchrzak, A. Cichoń, S. Borucki, 42, 29-35 (2017)

5. T. Boczar, D. Zmarzły, Insight 45, 488-492 (2003)

6. S. Krzewiński, P. Frącz, I. Urbaniec, T. Turba, J. Spectro. 2017 (2017)

7. P. Frącz, I. Urbaniec, T. Turba, S. Krzewiński, J. Spectro. 2016 (2016)

8. A. Reid, M. Judd, C. Johnstone, UPEC 44, 1-4 (2009)

9. M. Siegel, M. Beltle, S.Tenbohlen, S. Coenen, IEEE Trans. Dielectr. Electr. Insul. 24, 331-339 (2017)

10. J. Gielniak, A. Graczkowski, H. Moranda, P. Przybylek, K. Walczak, Z. Nadolny, H. MościckaGrzesiak, IEEE Trans. Dielectr. Electr. Insul. 20, 982-987 (2013)

11. J. Song, M. Wen, L. Li, A. Isenberg, IEEE Int. Conf. on Dielectr. 2, 625-629 (2016)

12. S. Wolny, M. Lepich, IEEE Int. Conf. on Dielectr. 2, 1163-1166 (2016)

13. T. Suwnansri, iEECON, 1-4 (2014) 